Original Article

\title{
Reliability of sagittal vertical axis measurement and association with measures of age-related hyperkyphosis
}

\author{
Wendy B Katzman, PT, DPTSc ${ }^{1{ }^{*}}$, Neeta Parimi, MS ${ }^{2)}$, Amy Gladin, PT, DPT ${ }^{3)}$, Bo Fan, MD ${ }^{4}$, \\ Shirley S Wong, BS ${ }^{1)}$, Joncarmen Mergenthaler, BS ${ }^{5)}$, Nancy E Lane, MD ${ }^{6)}$ \\ 1) Department of Physical Therapy and Rehabilitation Science, University of California, \\ San Fransisco: 1500 Owens St., Ste 400, San Francisco, CA 94158, USA \\ 2) San Francisco Coordinating Center, USA \\ 3) Kaiser Permanente Northern CA, USA \\ 4) Department of Radiology and Biomedical Imaging, University of California, San Francisco, USA \\ 5) Department of Neurological Surgery, University of California, San Francisco, USA \\ 6) University of California, Davis, USA
}

\begin{abstract}
Purpose] Persons with age-related hyperkyphosis often have concomitant sagittal plane imbalance of the spine. This study investigated the reliability of sagittal vertical axis (SVA) measurement of sagittal balance, association between thoracic Cobb angle of kyphosis and SVA measure of sagittal balance, and compared the degree of SVA in males and females with age-related hyperkyphosis. [Participants and Methods] Measurements of SVA and Cobb angle of kyphosis were obtained from baseline radiographs of 112 community-dwelling males and females, mean age 70.0 ( $\mathrm{SD}=5.7$ ) years with kyphosis $\geq 40$ degrees, recruited for a randomized controlled trial. Spearman correlation coefficients were used to determine associations between SVA and kyphosis, and Wilcoxon nonparametric tests to compare SVA between genders. [Results] SVA was acquired with excellent intra-rater [0.95 (95\% CI: 0.88, 0.98)] and inter-rater reliability [0.93 (95\% CI: 0.83,0.97)]. There was no significant correlation between Cobb angle of thoracic kyphosis and SVA, $(\mathrm{r}=-0.05)$. More males than females had sagittal imbalance (SVA $\geq 5 \mathrm{~cm}$ ). [Conclusion] In older adults with hyperkyphosis, SVA was a reliable measure of sagittal balance, and more extreme in males. SVA was not associated with Cobb angle of thoracic kyphosis, and could be considered an independent phenotype of age-related hyperkyphosis to be targeted in future intervention trials. Key words: Sagittal balance, Sagittal vertical axis, Hyperkyphosis
\end{abstract}

(This article was submitted Jul. 13, 2018, and was accepted Sep. 12, 2018)

\section{INTRODUCTION}

Measurement of thoracic kyphosis in persons with age-related hyperkyphosis is important for quantifying the degree of kyphosis deformity of the spine, and monitoring its progression and response to targeted interventions. The gold standard method of measuring hyperkyphosis is standing radiographic Cobb angle of kyphosis from T5 to T12 ${ }^{1,2)}$. However, other methods are often used to quantify hyperkyphosis, including the Block method ${ }^{3)}$, and occiput to wall ${ }^{4)}$ that quantify the position of the head in relation to the pelvis, aspects of sagittal balance, rather than the kyphosis deformity alone ${ }^{5)}$. Persons with age-related hyperkyphosis who have sagittal imbalance of the spine may be at increased risk for vertebral fractures and incident falls $\mathrm{s}^{6,7)}$ because of the altered distribution of body mass and the biomechanical environment of the spine when the head position is anterior to the pelvic sacral promontory ${ }^{6}$. Furthermore, when Cobb angle is measured globally (from T1 to

*Corresponding author. Wendy Katzman (E-mail: Wendy.Katzman@ucsf.edu)

(C2018 The Society of Physical Therapy Science. Published by IPEC Inc.

(c) (1) $\odot$ This is an open-access article distributed under the terms of the Creative Commons Attribution Non-Commercial No DerivaCC BY NC ND tives (by-nc-nd) License. (CC-BY-NC-ND 4.0: https://creativecommons.org/licenses/by-nc-nd/4.0/) 
T12) it can be affected by endplate tilt of the upper and lower vertebrae of the thoracic curvature, which may increase the magnitude of the curve but may not reflect changes in the curve relative to vertical alignment ${ }^{8}$. While studies have reported significant adverse health outcomes among those with thoracic kyphosis $\geq 40$ degrees $^{1,2)}$, global sagittal balance of the spine has been described as the most significant radiographic parameter predicting clinical symptoms in adult spinal deformity ${ }^{9,10)}$, making it an important target for future intervention.

Previous research suggests gender differences in the prevalence of age-related hyperkyphosis depending upon the methods used to quantify hyperkyphosis. Approximately $18 \%$ of males and $30 \%$ of females were categorized as hyperkyphotic in a cohort 70-80 year olds when a supine 40-degree thoracic kyphosis measurement was used to define hyperkyphosis ${ }^{11)}$. In contrast, when the supine Block method was used, $46 \%$ of males and $22 \%$ of females were categorized as hyperkyphotic in a similarly aged cohort ${ }^{12}$. Given the large variability in prevalence estimates for hyperkyphosis in males and females when different methods are used to quantify kyphosis, it is possible that males and females have different phenotypes of hyperkyphosis. Males may have more sagittal imbalance, and females may have more sagittal deformity of thoracic kyphosis. Therefore, the aims of this study were to determine the reliability of sagittal vertical axis measurement (SVA) of sagittal balance in community-dwelling asymptomatic older adults with hyperkyphosis $\geq 40$ degrees. We also investigated the association between thoracic kyphosis deformity and SVA measurement of sagittal balance, and compared the degree of sagittal balance in males and females with age-related hyperkyphosis. If thoracic kyphosis deformity and sagittal spine imbalance are independent phenotypes of hyperkyphosis, interventions to reduce age-related hyperkyphosis should be designed to improve both thoracic kyphosis deformity and sagittal balance.

\section{PARTICIPANTS AND METHODS}

We included 112 participants, mean age 70.0 ( $\mathrm{SD}=5.7)$ years, who were recruited January 2013 through June 2015 from local senior centers and outpatient medical clinics at two large urban medical centers (a university-based center and an integrated managed-care center), and enrolled in the Specialized Center of Research (SCOR) Kyphosis Project, a randomized controlled trial to investigate gender differences in response to a targeted kyphosis exercise intervention. All participants were screened for kyphometer-derived kyphosis $\geq 40$ degrees and age $\geq 60$ years as inclusion criteria for the SCOR Kyphosis Project. Participants were excluded for inability to straighten the thoracic spine at least five degrees, cognitive impairment ${ }^{13)}$, inability to pass safety tests in the screening examination, or any disorder or disease likely to interfere with safe participation in a group-based exercise class ${ }^{14)}$.

Protocols were approved by the University of California San Francisco (IRB No. 12-09348) and Kaiser Permanente Northern California (IRB No. CN-13AGlad-01-H) IRBs and written informed consent was collected for each participant.

Cobb angle measurements of kyphosis from lateral spine radiographs acquired at the baseline enrollment visit were used. Standing lateral spine radiographs were acquired using a standardized sagittal view scoliosis protocol from T3 to $\mathrm{S} 1$, with arms at a 90-degree angle to the body to prevent superimposition of the vertebrae, knees straight, and upon full inspiration. The degree of kyphosis was calculated by an experienced musculoskeletal radiologist (BF) using digitized Cobb angle derived from the radiographs scans centered at T8 (T4-T12). The Cobb method was used to determine the superior and inferior margins for line placement. Using a translucent digitizer (GTCO, Rockville, MD, USA) and cursor, the reader marked points corresponding to the four corners of the vertebral body at T4 and T12. From the superior surface of T4 and the inferior surface of $\mathrm{T} 12$, along the endplates of the vertebrae, a digitization program erected perpendicular lines, the intersection of which is Cobb angle (Fig. 1). The intra-rater reliability ICC for repeated digitized Cobb angle readings was previously reported ICC $=0.99^{15}$ ). SVA measurements of sagittal balance were acquired from the same standing lateral spine radiographs by an experienced musculoskeletal radiologist (BF). SVA was identified as the location of the head with respect to the normal center of gravity by a plumb line dropped from the center of the $\mathrm{C} 7$ vertebral body to the posterior superior corner of the sacral end plate ${ }^{16)}$. A vertical line drawn from the vertebral body of $\mathrm{C} 7$ normally intersects the superior endplate of S1. The horizontal distance of displaced SVA from a reference point on the sacral end plate was determined (Fig. 2). Repeat measurements were performed on a random sample of $15 \%$ of the scans by a second observer (JM) to determine the intraobserver and interobserver reliability for the SVA measurement.

Additional measurements of thoracic kyphosis and lumbar lordosis were acquired using kyphometer-derived thoracic kyphosis and lumbar lordosis measurements, and radiographic centroid measurement of thoracic kyphosis. The kyphometer angle of kyphosis was measured as the angle formed by the 2 arms of a Debrunner kyphometer (Proteck AG, Berne, Switzerland) protractor-device placed over the spine. The base of the top arm was aligned over the T2-3 interspace, and the base of the lower arm was aligned over the T11-T12 interspace. Similarly, for lumbar lordosis, the kyphometer angle of lordosis was measured as the angle formed by the arms of the device placed over the T11-T12 interspace and the L4-L5 interspace. All kyphometer measures were acquired by a trained exercise physiologist at the UCSF Clinical Research Center. For the radiographic centroid angle method, we used intersecting diagonals between two vertebral corners at both ends of the thoracic spine curve. The centroid curvature angle was defined as the angle between the straight lines drawn perpendicular through the two top and two bottom vertebrae (Fig. 3). Reliability of these methods has been previously established ${ }^{8,15)}$.

Other study covariates: Age and gender were determined at enrollment.

Statistical analysis: Characteristics including age, gender, and all spinal variables were summarized by descriptive statis- 


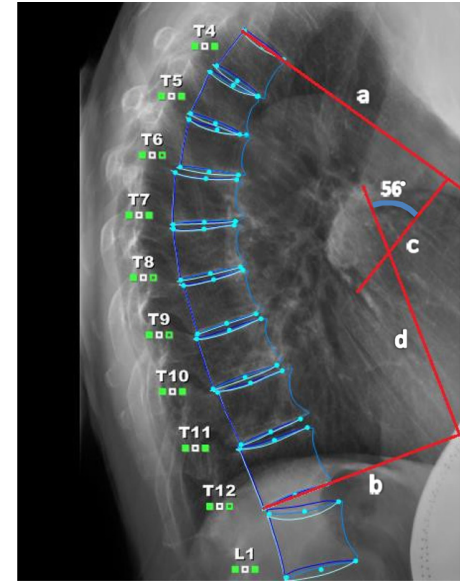

Fig. 1. Cobb angle of kyphosis measured from standing lateral radiograph. Line a is drawn from the superior endplate of T4; line $b$ is drawn from the inferior endplate of T12; lines $\mathrm{c}$ and $\mathrm{d}$ are perpendicular lines drawn from lines $a$ and $b$. Cobb angle of kyphosis is where lines $c$ and $d$ intersect.

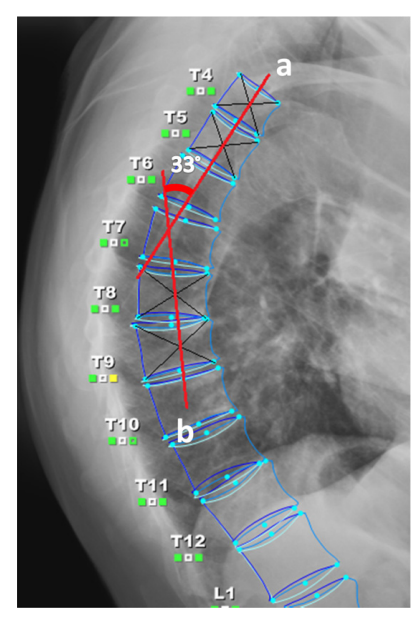

Fig. 3. Centroid angle was determined by measuring the intersection angle of two lines, which passed through T4T5 (a) centroid points and T8-T9 centroid points (b).

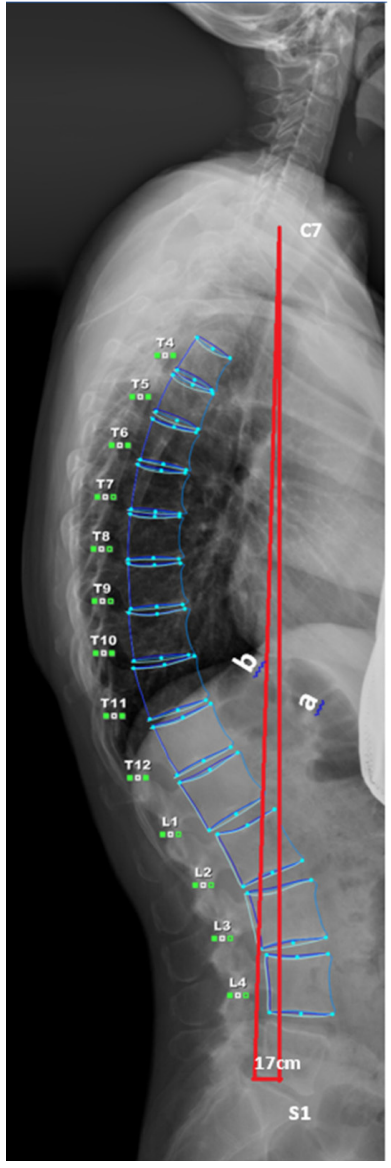

Fig. 2. The sagittal vertical axis is measured as the horizontal distance between a plumb line drawn from center of C7 (a) and a line drawn from center of C7 to posterior superior corner of S1 (b).

tics and compared between males and females using t-test or Wilcoxon nonparametric test for continuous variables and the Fisher exact or $\chi^{2}$ test for categorical variables. Nonparametric counterparts were used for variables that were not normally distributed, based upon testing for kurtosis value $<-2$ and $>+2^{17}$ ). We used intra-class correlation coefficients (ICC) with 95 percent confidence intervals $(95 \% \mathrm{CI})$ to compare the relative intra- and inter-rater reliability of SVA measurement. Two readers $(\mathrm{BF}, \mathrm{JM})$ blinded to each other's results, read a random sample of 15 films, and each reader read 15 films twice after a month interval. To evaluate the strength, of the correlation, we used a method outlined by Munro (0.9-1.0="very high"; $0.7-0.89=$ "high"; $0.5-0.69=$ "moderate"; $0.26-0.49=$ "low"; $0.0-0.25=$ "little if any") ${ }^{18)}$. We assessed the absolute reliability of the SVA measurements and calculated the standard error of the measurement [(SEM=standard deviation of sagittal plane measurement* square root of $(1-\mathrm{ICC})]$ and minimal detectable change $\left[\mathrm{MDC}=1.96 * \mathrm{SEM}^{*} \text { square root of }(2)\right]^{19)}$. We used Spearman correlation coefficients to determine the association between Cobb angle, SVA and other measures of hyperkyphosis, and considered correlations ranging from 0.00 to 0.25 to indicate little or no relationship; 0.25 to 0.5 a fair relationship; 0.5 to 0.75 moderate to good; and values above 0.75 good to excellent ${ }^{20)}$. We used Wilcoxon nonparametric tests for continuous variables to compare SVA in both genders. Kyphometer-measured kyphosis, and lumbar lordosis were included to investigate concurrent validity of the radiographic and clinical measures of hyperkyphosis. Centroid measure of kyphosis 
was included to account for endplate tilt. We also categorized SVA as quartiles and assessed the distribution of quartiles of SVA between genders. All analyses were performed with SAS 9.4 (Cary, NC, USA).

\section{RESULTS}

Characteristics of our study participants, 45 males and 67 females, are presented in Table 1. Age ranged from 60 to 92 , with an average age of $70.0(\mathrm{SD}=5.7)$ years old. Mean Cobb angle was 55.6 ( $\mathrm{SD}=12.1)$ degrees, with no difference between genders. SVA measurements were acquired on all but 2 of the scans where visualization of $\mathrm{C} 7$ vertebral body superiorly was not possible. The intra-rater reliability of the SVA measurement was ICC $=0.95$ (95\%CI: $0.88,0.98)$. The inter-rater reliability was ICC $=0.93$ (95\%CI: 0.83, 0.97). The within and between subject SEM was 7.7 and $9.15 \mathrm{~mm}$, and MDC was 21.4 and $25.4 \mathrm{~mm}$, respectively.

SVA measurement was skewed in males, although the median SVA in males compared to females was not statistically different, $\mathrm{p}=0.35$ (Table 1). The kurtosis value was 1.7 overall. When we used $5 \mathrm{~cm}$ or more of SVA as a threshold for abnormal $^{21)}$, more males than females, $n=12(26.7 \%)$ versus $n=5(7.5 \%)$, were classified with abnormal SVA, and the difference was significant, $\mathrm{p}=0.006$. There were no differences in centroid angle in males and females, but males had greater kyphometer-derived kyphosis, $53.3(\mathrm{SD}=7.2)$ vs. $51.3(\mathrm{SD}=7.5)$ degrees, $\mathrm{p}=0.06$. Males also had less lordosis than females, $23.1(\mathrm{SD}=11.1)$ vs. $34.9(\mathrm{SD}=10.3)$ degrees, $\mathrm{p}<0.001$. There were no differences between males and females in the prevalence estimates for hyperkyphosis when Cobb angle $\geq 40$ degrees was used, however, a greater proportion of males were categorized as hyperkyphotic when Cobb angle $\geq 54$ degrees or SVA $\geq 5 \mathrm{~cm}^{\text {thresholds were used }}{ }^{7,10)}$.

Using Cobb angle of kyphosis as the gold standard, there was no significant association with sagittal balance measurement of SVA (r:-0.05, p=0.59) (Table 2). There was a strong association with the centroid angle $(\mathrm{r}: 0.59, \mathrm{p}<0.001)$ and kyphometer-derived kyphosis ( $\mathrm{r}:-0.58, \mathrm{p}<0.001)$. The association of Cobb angle of kyphosis and lumbar lordosis was weak $(\mathrm{r}:-0.40, \mathrm{p}<0.001$, and lumbar lordosis and SVA were inversely correlated (r: $-0.19, \mathrm{p}=0.04)$. More males than females, 15 $(33.3 \%)$ versus $13(19.4 \%)$, were categorized with the most extreme sagittal balance (Table 3$)$. However, when the upper quartile of SVA ( $\geq 35.5$ degrees) was compared to the lower 3 quartiles, the $p$-value was not significant, $p=0.095$.

\section{DISCUSSION}

In older community-dwelling males and females with kyphosis $\geq 40$ degrees, we found that SVA can be measured with high reliability, which makes this an appealing, objective measurement of sagittal balance. In our cohort of older adults with hyperkyphosis (mean radiographic Cobb angle 55.6 degrees), there was no significant association between the gold-standard Cobb angle measure of thoracic kyphosis deformity and the SVA measure of sagittal balance. These findings highlight the difference in thoracic kyphosis deformity and sagittal balance, and suggest that the SVA quantifies an independent, distinct phenotype of hyperkyphosis. In contrast, Cobb angle of kyphosis was associated with other spinal deformity measures including radiographic centroid angle of kyphosis and non-radiographic kyphometer-derived kyphosis and lumbar lordosis, and supports the measurement of these conditions as methods for quantifying distinct aspects of hyperkyphosis. Moreover, more males were classified with abnormal SVA when the threshold of $5 \mathrm{~cm}$ or more SVA was used, which puts them at greater risk for disability ${ }^{10)}$.

We found that SVA is a highly reproducible measurement, consistent with a previous study that reported intra- and interrater reliability $\left(\mathrm{ICC}=0.92\right.$ and 0.995 respectively) ${ }^{22}$. Results of our study also provide a distribution-based MDC estimate that may be useful to estimating reliable change in SVA in future studies. However it is important to recognize that Van Royen et al. ${ }^{23)}$ examined reproducibility of SVA using digital markers in a man with ankylosing spondylitis and a fixed spine, and found that small changes in the hip, knee, and ankle joints affected the SVA measure. Hayden et al. also reported that both anterior and posterior rotation of the pelvis ${ }^{24)}$ can significantly alter spinopelvic parameters including SVA. To reduce variability in the radiographic image and compensatory means to restore sagittal balance and maintain horizontal gaze, our protocol was strictly defined by stance in usual posture with "knees straight", arms supported at 90 degrees of flexion and respiration suspended upon full inhalation. Moreover, our SVA measures were obtained from repeat measures from the same radiograph; variable results could have been possible had we used multiple different radiographs.

We found no association between standing radiographic measurements of Cobb angle of kyphosis and SVA. This null finding is consistent with a recent study that reported little or no association between standing radiographic Cobb angle and SVA, $r=0.12$, in a cohort of 1,461 participants mean age $66.3 \pm 13.8$ years for males and $65.2 \pm 12.5$ years for females ${ }^{25}$. In contrast, Tran et al. ${ }^{3)}$ reported a moderate to good association between the supine non-radiographic block method measurement of kyphosis, which captures the forward head component of hyperkyphosis while lying in a supine position, and a supine radiographic measurement of Cobb angle, $r=0.63$. Moreover, neither one of the supine measures are influenced by postural effects of gravity on the spine, which points to the influential effects of gravity on thoracic kyphosis and sagittal balance ${ }^{26)}$.

Our results suggest that the method used to quantify hyperkyphosis can produce very different prevalence estimates. When we used a hyperkyphosis threshold of Cobb angle $\geq 40$ degrees, $88 \%$ of our cohort was classified as hyperkyphotic. At a 54 degree threshold, $39 \%$ of the cohort was classified as hyperkyphotic, consistent with previous reports of $20-40 \%$ prevalence 
Table 1. Participant characteristics

\begin{tabular}{|c|c|c|c|c|}
\hline & $\begin{array}{c}\text { Overall } \\
\left(\mathrm{N}=112^{*}\right)\end{array}$ & $\begin{array}{c}\text { Men } \\
\left(\mathrm{N}=45^{*}\right)\end{array}$ & $\begin{array}{c}\text { Women } \\
\left(\mathrm{N}=67^{*}\right)\end{array}$ & $\mathrm{p}$-value \\
\hline & & Mean \pm SD & & \\
\hline Age (years) & $70.0 \pm 6.2$ & $70.9 \pm 6.5$ & $69.3 \pm 6.0$ & 0.21 \\
\hline Cobb angle of kyphosis (degrees) & $55.6 \pm 12.1$ & $55.0 \pm 12.2$ & $55.9 \pm 12.1$ & 0.71 \\
\hline Centroid angle (degrees) & $33.6 \pm 9.1$ & $33.0 \pm 8.83$ & $33.9 \pm 9.3$ & 0.64 \\
\hline Kyphometer-derived kyphosis (degrees) & $52.1 \pm 7.4$ & $53.3 \pm 7.2$ & $51.3 \pm 7.5$ & 0.06 \\
\hline Kyphometer-derived lordosis (degrees) & $30.1 \pm 12.1$ & $\begin{array}{c}23.1 \pm 11.1 \\
\mathrm{~N}(\%)\end{array}$ & $34.9 \pm 10.3$ & $<0.0001$ \\
\hline Cobb angle $\geq 40$ degrees & $96(88.1)$ & $39(88.6)$ & $57(87.7)$ & 0.88 \\
\hline Kyphometer-derived kyphosis $\geq 54$ degrees & $44(39.3)$ & $24(53.3)$ & $20(29.9)$ & 0.01 \\
\hline Sagittal vertical axis $\geq 5 \mathrm{~cm}$ & $17(15.2)$ & $\begin{array}{c}12(26.7) \\
\text { Median (IQR) }\end{array}$ & $5(7.5)$ & 0.006 \\
\hline Sagittal vertical axis $(\mathrm{mm})$ & $17.2(-1.4$ to 84.6$)$ & $17.8(-1.5$ to 103.6$)$ & $16.7(-1.4$ to 54.9$)$ & 0.35 \\
\hline
\end{tabular}

Table 2. Correlations between sagittal vertical axis and measures of age-related hyperkyphosis

\begin{tabular}{|c|c|c|c|c|c|}
\hline & $\begin{array}{c}\text { Centroid angle } \\
\text { (degrees) }\end{array}$ & $\begin{array}{l}\text { Sagittal vertical axis } \\
(\mathrm{mm})\end{array}$ & $\begin{array}{l}\text { Cobb angle } \\
\text { (degrees) }\end{array}$ & $\begin{array}{r}\text { Lordosis } \\
\text { (degrees) }\end{array}$ & $\begin{array}{l}\text { Kyphosis } \\
\text { (degrees) }\end{array}$ \\
\hline Centroid angle (degrees) & 1 & -0.003 & $0.59^{\mathrm{a}}$ & $0.24^{\mathrm{b}}$ & $0.34^{\mathrm{a}}$ \\
\hline Sagittal vertical axis $(\mathrm{mm})$ & -0.003 & 1 & -0.05 & $-0.19^{\mathrm{b}}$ & 0.06 \\
\hline Cobb angle (degrees) & $0.59^{\mathrm{a}}$ & -0.05 & 1 & $0.40^{\mathrm{a}}$ & $0.58^{\mathrm{a}}$ \\
\hline Lordosis (degrees) & $0.24^{\mathrm{b}}$ & $-0.19^{b}$ & $0.40^{\mathrm{a}}$ & 1 & 0.11 \\
\hline Kyphosis (degrees) & $0.34^{\mathrm{a}}$ & 0.06 & $0.58^{\mathrm{a}}$ & 0.11 & 1 \\
\hline
\end{tabular}

Table 3. Number (\%) of men and women by quartile of sagittal vertical axis

\begin{tabular}{ccccc}
\hline & & Men $(\mathrm{N}=45)$ & Women $(\mathrm{N}=65)$ & p-value \\
& $\mathrm{N}(\%)$ & $\mathrm{N}(\%)$ & \\
\hline Low: & $-60.0 \leq \mathrm{Q} 1<-1.4 \mathrm{~mm}$ & $12(27)$ & $16(24)$ & 0.74 \\
& $-1.4 \leq \mathrm{Q} 2<17.2 \mathrm{~mm}$ & $10(22)$ & $18(27)$ & 0.58 \\
& $17.2 \leq \mathrm{Q} 3<35.5 \mathrm{~mm}$ & $8(18)$ & $20(30)$ & 0.15 \\
High: & $35.5 \leq \mathrm{Q} 4<154.2 \mathrm{~mm}$ & $15(33)$ & $13(19)$ & 0.095 \\
\hline
\end{tabular}

in older adults. In contrast to previously published thresholds that describe SVA imbalance at $5 \mathrm{~cm}$ or more ${ }^{10,21)}$, only $15 \%$ of our cohort who had a mean Cobb angle of 55.6 \pm 12.1 degrees was classified with sagittal imbalance. Thus, these methods to measure hyperkyphosis cannot be used interchangeably because they quantify different phenotypes of hyperkyphosis.

Our study highlights differences between sagittal deformity and sagittal balance. When the sagittal curves of the spine and the pelvis balance each other, the spine is aligned in relation to the pelvis, and the horizontal gaze is intact ${ }^{27)}$. Once the sagittal balance is lost, more energy is required for the body to remain balanced without external support ${ }^{27)}$. According to Lamartina et al. ${ }^{27)}$, ideal sagittal balance can be predicted as a combination of regional deformities and compensatory mechanisms. When the expected or possible compensatory mechanisms do not occur, imbalance and abnormal muscle activation patterns develop. For example, Bruno et al. ${ }^{6)}$ investigated vertebral load in a hyperkyphosis computer model, and reported greater spinal load in the hyperkyphotic condition where the magnitude of kyphosis was increased. Load was further increased in the sagittal imbalance condition where the head aligned anterior to the pelvis. However, maintaining sagittal balance with kyphosis deformity effectively reduced vertebral loads, and highlights the independence of these sagittal spine subtypes.

Our study was conducted in a sample of older males and females large enough to investigate differences between males and females. However, SVA was skewed in males and this may have affected the results. Also, measurement of sagittal balance can be measured using the T1 pelvic angle defined as the angle formed by lines drawn from the center of the femoral 
head axis to the center of T1 vertebral body and to the middle of the S1 endplate ${ }^{28)}$, and sagittal balance is affected by pelvic parameters including pelvic rotation, pelvic incidence and sacral slope that were not measured in our study. The radiographs were acquired for assessment of Cobb angle of kyphosis and they did not include the pelvis. Regardless, our results may be viewed as a discrete but not exhaustive contribution to our understanding of age-related hyperkyphosis, spinal deformity, and sagittal balance. In older males and females with hyperkyphosis, SVA was a reliable measure of sagittal balance. Our study suggests that radiographic Cobb angle measure of thoracic kyphosis deformity and SVA measure of sagittal balance are independent and distinct phenotypes of age-related hyperkyphosis. Other measures that are often used to quantify age-related hyperkyphosis, including kyphometer-derived kyphosis and lordosis, and centroid measure of kyphosis were associated with the gold standard Cobb angle of thoracic kyphosis. Despite similar degrees of radiographic Cobb angle of thoracic kyphosis, males had more sagittal imbalance than females. Given the clinical importance of sagittal balance, future studies may be warranted to determine whether both thoracic kyphosis deformity and sagittal balance can be improved with targeted interventions.

ClinicalTrials.gov Identifier No.: NCT01766674

\section{Funding}

The study was funded by a pilot study grant from the NIH P50 AR063043 grant from the Office of Research in Women's Health and the National Institute of Arthritis and Musculoskeletal and Skin Disorders.

\section{Conflicts of interest}

None of the authors have conflict of interest.

\section{REFERENCES}

1) Balzini L, Vannucchi L, Benvenuti F, et al.: Clinical characteristics of flexed posture in elderly women. J Am Geriatr Soc, 2003, 51: 1419-1426. [Medline] [CrossRef]

2) Takahashi T, Ishida K, Hirose D, et al.: Trunk deformity is associated with a reduction in outdoor activities of daily living and life satisfaction in communitydwelling older people. Osteoporos Int, 2005, 16: 273-279. [Medline] [CrossRef]

3) Tran TH, Wing D, Davis A, et al.: Correlations among four measures of thoracic kyphosis in older adults. Osteoporos Int, 2016, 27: 1255-1259. [Medline] [CrossRef]

4) Antonelli-Incalzi R, Pedone C, Cesari M, et al.: Relationship between the occiput-wall distance and physical performance in the elderly: a cross sectional study. Aging Clin Exp Res, 2007, 19: 207-212. [Medline] [CrossRef]

5) Kado DM, Christianson L, Palermo L, et al.: Comparing a supine radiologic versus standing clinical measurement of kyphosis in older women: the Fracture Intervention Trial. Spine, 2006, 31: 463-467. [Medline] [CrossRef]

6) Bruno AG, Anderson DE, D’Agostino J, et al.: The effect of thoracic kyphosis and sagittal plane alignment on vertebral compressive loading. J Bone Miner Res, 2012, 27: 2144-2151. [Medline] [CrossRef]

7) McDaniels-Davidson C, Davis A, Wing D, et al.: Kyphosis and incident falls among community-dwelling older adults. Osteoporos Int, 2018, 29: 163-169. [Medline] [CrossRef]

8) Briggs AM, Wrigley TV, Tully EA, et al.: Radiographic measures of thoracic kyphosis in osteoporosis: Cobb and vertebral centroid angles. Skeletal Radiol, 2007, 36: 761-767. [Medline] [CrossRef]

9) Glassman SD, Bridwell K, Dimar JR, et al.: The impact of positive sagittal balance in adult spinal deformity. Spine, 2005, 30: 2024-2029. [Medline] [CrossRef]

10) Schwab FJ, Blondel B, Bess S, et al. International Spine Study Group (ISSG): Radiographical spinopelvic parameters and disability in the setting of adult spinal deformity: a prospective multicenter analysis. Spine, 2013, 38: E803-E812. [Medline] [CrossRef]

11) Katzman W, Cawthon P, Hicks GE, et al.: Association of spinal muscle composition and prevalence of hyperkyphosis in healthy community-dwelling older men and women. J Gerontol A Biol Sci Med Sci, 2012, 67: 191-195. [Medline] [CrossRef]

12) Kado DM, Huang MH, Nguyen CB, et al.: Hyperkyphotic posture and risk of injurious falls in older persons: the Rancho Bernardo Study. J Gerontol A Biol Sci Med Sci, 2007, 62: 652-657. [Medline] [CrossRef]

13) Borson S, Scanlan JM, Chen P, et al.: The Mini-Cog as a screen for dementia: validation in a population-based sample. J Am Geriatr Soc, 2003, 51: 1451-1454. [Medline] [CrossRef]

14) Katzman WB, Parimi N, Gladin A, et al.: Sex differences in response to targeted kyphosis specific exercise and posture training in community-dwelling older adults: a randomized controlled trial. BMC Musculoskelet Disord, 2017, 18: 509. [Medline] [CrossRef]

15) Lundon KM, Li AM, Bibershtein S: Interrater and intrarater reliability in the measurement of kyphosis in postmenopausal women with osteoporosis. Spine, 1998, 23: 1978-1985. [Medline] [CrossRef]

16) Hammerberg EM, Wood KB: Sagittal profile of the elderly. J Spinal Disord Tech, 2003, 16: 44-50. [Medline] [CrossRef]

17) George D. SPSS for windows step by step: A simple study guide and reference, 17.0 update, 10/e: Pearson Education India, 2011.

18) Plichta SB, Kelvin EA, Munro BH: Munro's statistical methods for health care research. Philadelphia: Wolters Kluwer Health/Lippincott Williams \& Wilkins; 2005, pp 239-258.

19) Haley SM, Fragala-Pinkham MA: Interpreting change scores of tests and measures used in physical therapy. Phys Ther, 2006, 86: 735-743. [Medline]

20) Watkins LP: Foundations of clinical research: applications to practice, 2nd ed. New Jersey: Prentice Hall Health, 2000.

21) Schwab F, Lafage V, Boyce R, et al.: Gravity line analysis in adult volunteers: age-related correlation with spinal parameters, pelvic parameters, and foot posi- 
tion. Spine, 2006, 31: E959-E967. [Medline] [CrossRef]

22) Protopsaltis T, Schwab F, Bronsard N, et al. International Spine Study Group: The T1 pelvic angle, a novel radiographic measure of global sagittal deformity, accounts for both spinal inclination and pelvic tilt and correlates with health-related quality of life. J Bone Joint Surg Am, 2014, 96: 1631-1640. [Medline] [CrossRef]

23) Van Royen BJ, Toussaint HM, Kingma I, et al.: Accuracy of the sagittal vertical axis in a standing lateral radiograph as a measurement of balance in spinal deformities. Eur Spine J, 1998, 7: 408-412. [Medline] [CrossRef]

24) Hayden AM, Hayes AM, Brechbuhler JL, et al.: The effect of pelvic motion on spinopelvic parameters. Spine J, 2018, 18: 173-178. [Medline] [CrossRef]

25) Asai Y, Tsutsui S, Oka H, et al.: Sagittal spino-pelvic alignment in adults: the Wakayama spine study. PLoS One, 2017, 12: e0178697. [Medline] [CrossRef]

26) Yokoyama Y, Nishiwaki Y, Michikawa T, et al.: The association of kyphosis assessed in supine and standing positions with future activities of daily living dependence: the Kurabuchi Study. Arch Osteoporos, 2017, 12: 105. [Medline] [CrossRef]

27) Lamartina C, Berjano P: Classification of sagittal imbalance based on spinal alignment and compensatory mechanisms. Eur Spine J, 2014, 23: 1177-1189. [Medline] [CrossRef]

28) Plachta SM, Israel H, Brechbuhler J, et al.: Inter/intra-observer reliability of T1 Pelvic Angle (TPA), a novel radiographic measure for global sagittal deformity. Spine, 2018. [Medline] [CrossRef] 